\title{
Mutually Enhancing Community Detection and Sentiment Analysis on Twitter Networks
}

\author{
William Deitrick, Wei Hu* \\ Department of Computer Science, Houghton College, Houghton, USA \\ Email: *wei.hu@houghton.edu
}

Received June 3, 2013; revised July 5, 2013; accepted July 29, 2013

Copyright (C) 2013 William Deitrick, Wei Hu. This is an open access article distributed under the Creative Commons Attribution License, which permits unrestricted use, distribution, and reproduction in any medium, provided the original work is properly cited.

\begin{abstract}
The burgeoning use of Web 2.0-powered social media in recent years has inspired numerous studies on the content and composition of online social networks (OSNs). Many methods of harvesting useful information from social networks' immense amounts of user-generated data have been successfully applied to such real-world topics as politics and marketing, to name just a few. This study presents a novel twist on two popular techniques for studying OSNs: community detection and sentiment analysis. Using sentiment classification to enhance community detection and community partitions to permit more in-depth analysis of sentiment data, these two techniques are brought together to analyze four networks from the Twitter OSN. The Twitter networks used for this study are extracted from four accounts related to Microsoft Corporation, and together encompass more than 60,000 users and 2 million tweets collected over a period of 32 days. By combining community detection and sentiment analysis, modularity values were increased for the community partitions detected in three of the four networks studied. Furthermore, data collected during the community detection process enabled more granular, community-level sentiment analysis on a specific topic referenced by users in the dataset.
\end{abstract}

Keywords: Community Detection; Sentiment Analysis; Twitter; Online Social Networks; Modularity; Community-Level Sentiment Analysis

\section{Introduction}

The popularity of online social networks (OSNs) has increased dramatically in recent years. Individuals and organizations can now take advantage of a wide array of Web 2.0-powered social networking platforms, including the likes of Facebook, LinkedIn, and Twitter [1]. Though these services vary greatly in both form and function, they are all alike in facilitating the exchange of signifycant volumes of information among their users. Due to the massive amounts of data that flow through social networks and the relative ease of accessing this data, analysis of social networks has become a research topic of particular interest.

While the study of social networks can take many forms, two popular topics are community detection and sentiment analysis. Distinct groups of entities, or communities, will often form within social networks. Identifying these groups and modeling their dynamic interacttions can provide valuable insight across many disciplines, and is the primary goal of community detection

"Corresponding author.
[2]. Sentiment analysis, sometimes called opinion mining, provides a means of automatically determining the attitudes or opinions of users via the content they have created [3]. Using sentiment analysis and community detection techniques, previous research has demonstrated the usefulness of information gained from social networks in describing such real-world events and issues as political movements [4], elections [5], and consumer attitudes towards products and services [6]. In this study, both sentiment analysis and community detection will be used to analyze networks from the Twitter OSN.

\subsection{The Twitter OSN}

Since its inception in 2006, Twitter's overwhelmingly rapid growth has made this service the Internet's fastest growing social networking platform [1]. As a result, Twitter has become a popular target for research efforts. The service's powerful and well-documented Application Programming Interface (API) provides an easy means to obtain content created by Twitter users, and a plethora of libraries exist for many platforms and programming lan- 
guages to further simplify the data collection process.

Twitter is primarily a microblogging service, allowing its users to post, or "tweet" messages up to 140 characters in length. These messages often contain links to other web content in the form of URLs (usually abbreviated via URL shortening services) and Twitter-specific constructs including hashtags, mentions, replies, and retweets. Hashtags are words or terms denoting a specific topic preceded by the \# (pound) symbol, while mentions and replies are a means of referencing other Twitter users by prepending their username with the @ symbol in a message [1]. A retweet allows a user to re-post a tweet created by another user, usually indicating their support for or interest in that tweet's content. Twitter users can "follow" other individuals to receive the messages those users post to Twitter, and anyone following a particular user is denoted as a "follower" of that user [7]. By default, users' Tweets are publicly accessible, allowing Twitter users open access to each other's content via Twitter's web portal or its API [6].

\subsection{Community Detection}

Community detection is undoubtedly one of the most popular research topics associated with OSNs. To facilitate community detection, social networks are modeled as mathematical graphs, often referred to as "social graphs", in which vertices (or nodes) represent actors within the network and edges correspond to ties between individuals. Such a network is shown in Figure 1. In this image, the coloring of vertices represents possible communities to which each of the nodes in the network may belong. Depending on a network's purpose and the nature of the data being analyzed, its edges may be

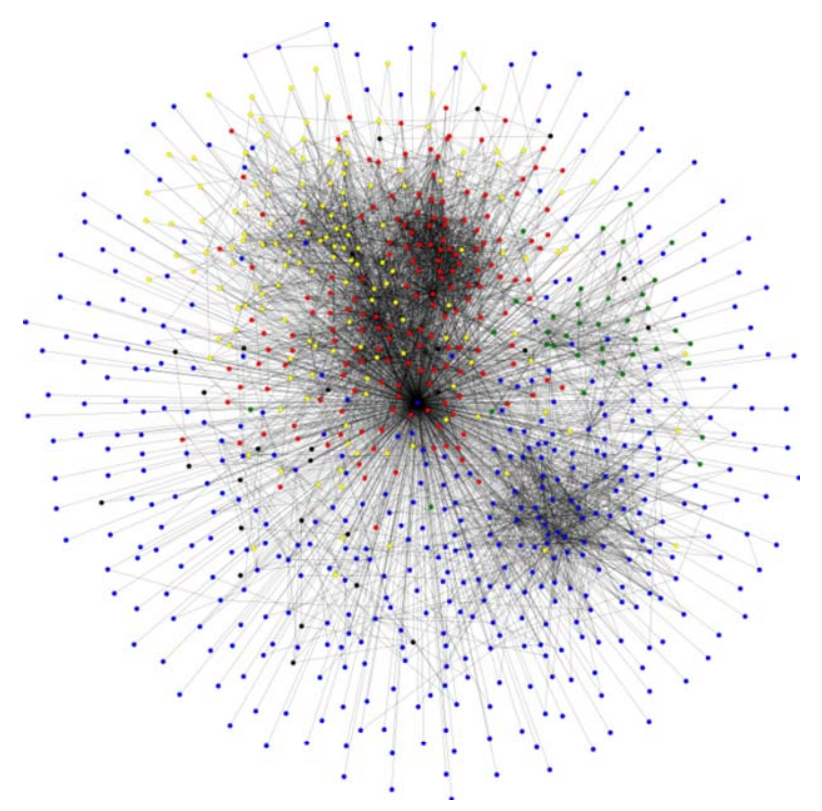

Figure 1. An example social network. weighted or unweighted as well as directed or undirected [8].

Communities of individuals in a social network can be distinguished using graph clustering techniques. The basic idea of graph clustering is to group similar or associated nodes together. Generally, partitions are created that maximize the number of connections (edges) within a cluster while minimizing connections between clusters [8]. Using graph clustering, previous studies have been able to effectively discover real-world communities on the Twitter OSN such as the Indie Mac developer community studied in [7].

Many graph clustering methods have been applied to community detection on social networks. Hierarchical clustering utilizes hierarchical representations of graphs called dendrograms. These structures provide easy control over clustering resolution, since each level of the hierarchy is effectively a clustering of the graph at a different level of granularity [8]. Though this is a popular method, its shortcomings have stimulated interest in other ideas. One alternative technique, proposed by Newman and Girvan, uses a measure called "betweenness” to discover clusters within a graph structure [9]. Betweenness is a metric applied to the edges within a graph and is defined as the number of shortest paths connecting any two nodes that pass through a given edge [8]. Algorithms based on optimizing edge betweenness that perform well on both real and computer-generated networks have been successfully developed [9]. Another algorithm, known as DENGRAPH, is a density-based clustering algorithm proposed specifically to analyze social network structures. Based on the incremental version of the DBSCAN algorithm, DENGRAPH provides a key asset in the study of social networks: the ability to handle the constant, dynamic changes in their structure [10]. Still another technique, known as the Label Propagation Algorithm (LPA), is based on the idea of a spreading disease epidemic. With this method, each node in the network is initially assigned a unique label. For every iteration after the initial step, each node is updated to have the label held by the majority of its neighbors (in the case of a tie, a label is picked at random). The effectiveness of this algorithm has been shown by several studies [11]. These examples are but a small sampling of the myriad community detection algorithms that have been proposed over the past few years.

One key aspect of community detection is quantifying the quality, or fitness, of the communities found. Indeed, many community detection algorithms are driven by optimization of one metric or another, such as the wellknown modularity metric [8]. Modularity, proposed by Newman and Girvan in [12], measures the fraction of edges within communities minus the expected value in a network with equivalent partitions but random edges. This quantity can be expressed as follows: 


$$
Q=\sum_{i=1}^{k}\left[\frac{e_{i}}{m}-\left(\frac{d_{i}}{2 m}\right)^{2}\right],
$$

where $k$ denotes the number of modules (partitions) created from the network, $e_{i}$ the number of edges in a module $i, d_{i}$ the sum the degrees of nodes in a module $i$, and $m$ the number of edges in the entire network [13]. Modularity can also take edge weights into account with just a slight change to the formula. Despite the popularity of this measure, it has been criticized for such issues as a significant resolution limit [14], stemming from the fact that modularity is oriented towards global optimization [15].

Another method for calculating community fitness, proposed in [16], simply measures the ratio of the internal degree of a community to the total degree of that community:

$$
f_{G}=\frac{k_{\text {in }}^{G}}{\left(k_{\text {in }}^{G}+k_{\text {out }}^{G}\right)^{\alpha}},
$$

where $k_{\text {in }}^{G}$ and $k_{\text {out }}^{G}$ denote the internal and external degrees of the nodes in a module $G$. The exponent $\alpha$ controls the size of the communities, and ought to be a positive real-valued number [16]. Both modularity and the degree ratio metric will be used in this study to evaluate the results of community detection. These are but two examples of the many methods for evaluating community structures in networks, and further reading on this topic can be found in [17].

In this study, two algorithms were chosen for community detection: the Speaker-Listener Label Propagation Algorithm (SLPA) [18] and the Infomap algorithm [19]. These were chosen because they are able to handle both weighted and directed networks, they both execute relatively quickly on large graphs, and because their operation differs greatly. SLPA is based on the Label Propagation Algorithm (LPA), but offers a critical extension: whereas in LPA each node may contain only a single label, SLPA allows each node to assume multiple labels. Thus, SLPA is able to detect overlapping communities, such as those that occur in a social network [18]. The implementation of SLPA used for this study was obtained from the creators of this algorithm at their website (https://sites.google.com/site/communitydetectionslpa/).

The Infomap algorithm, on the other hand, models information flow in a network using the probabilities of particular random walks within the network. This algorithm was designed for use with biological and sociological networks, and was originally demonstrated on a citation network of publications from the sciences [19]. The iGraph library (http://igraph.sourceforge.net) contains an implementation of this algorithm, which was used with the provided Python interface.

\subsection{Sentiment Analysis}

In addition to community detection, sentiment analysis has become another popular tool for analysis of social networks. Sentiment analysis is often formulated as a two-step problem, in which it is first necessary to determine whether a given text is subjective or objective. This is known as Subjective/Objective-polarity, or SO-polarity. If it is determined that a text is subjective, it can then be classified according to whether it expresses positive or negative sentiment, which is denoted Positive/Negativepolarity or PN-polarity [20]. Over the past few years, a variety of strategies have been used to perform sentiment analysis on many different types of data.

A common approach to sentiment analysis uses a lexicon of words labeled with their SO or PN polarities. The SentiWordNet lexicon [20] is one such resource that has proven effective in analyzing all manner of text documents, from product reviews [21] to news headlines [22], and has even been used for multilingual sentiment analysis [23]. When using a lexicon such as SentiWordNet, a simple technique involves summing the polarity scores for the words in a document and making a prediction based on the result. While this naïve approach can produce satisfactory results, lexicon-based polarity scores can be used more accurately when coupled with a machine learning algorithm [24].

While lexicon-based methods have been shown to be effective for many types of textual documents, Twitter presents a unique challenge because its informal messages are very short and contain large amounts of slang and misspellings. This reduces the effectiveness of traditional lexicons [3]. As a result, some studies such as [3] and [25] have chosen to use fully or distantly supervised learning to more accurately classify the sentiment expressed in tweets. Fully supervised learning requires manually labeling data to provide input for a machine learning classifier. This is a useful technique, but is extremely time-consuming and often produces training sets that are not of sufficient size to effectively train a classifier [3]. Distantly supervised methods attempt to overcome these issues by automatically labeling training datasets based on "noisy" labels such as emoticons and hashtags [26].

For this study, we adopt the fully supervised training approach using multiple datasets to ensure we have a training corpus of sufficient size. The Naïve Bayes classifier from the Natural Language Toolkit (http://nltk.org/), abbreviated NLTK, is used in conjunction with both unigram (single-token) and bigram (two-token) features to identify the subjective/objective and positive/negative orientation of tweets.

\subsection{Purpose of This Research}

While previous studies using sentiment analysis and 
community detection abound, these tasks are generally treated as completely separate issues. This study instead combines the two techniques, investigating the integration of sentiment analysis and community detection on networks from the Twitter OSN. Sentiment analysis is used in addition to other Twitter-specific features including hashtags, mentions, replies, and retweets to enhance community detection. Once community structures have been discovered, the power of combining these techniques is demonstrated by analyzing sentiment information on the community level in one of the dataset's networks.

\section{Datasets}

Two datasets were used in this study. The first of these was the publicly available Sanders corpus

(http://www.sananalytics.com/lab/twitter-sentiment/), a manually-labeled dataset provided for training sentiment classifiers. The second dataset was collected directly from the Twitter API for this study, and contained data relating to several Twitter accounts managed by Microsoft Corporation.

\subsection{Sanders Corpus}

The Sanders corpus consists of 5513 tweets manually labeled according to their subject and sentiment. The Twitter API terms of service do not permit direct distribution of the tweets, so a small Python script is provided to download the dataset directly from Twitter. While this corpus contains over 5500 tweets, some of the tweets appear to be no longer available via the Twitter API and could not be downloaded. Thus, the number of available tweets was reduced to 4957. Furthermore, only 3727 of the tweets are labeled according to sentiment as "positive", "negative”, or "neutral", while the rest are labeled "irrelevant". All of the tweets labeled "irrelevant" were filtered out, as well as those that were not English (according to the "lang" attribute provided by the Twitter API). Thus, the usable data for this study from the Sanders corpus contained 3111 tweets.

From the 3111 tweets extracted from the Sanders corpus, two datasets were created, and later merged with data from the Microsoft dataset described below. The first dataset was used in the training of a subjective/objective Naïve Bayes classifier. All "neutral” tweets were assigned the label "objective", and all positive and negative tweets were assigned the label "subjective". The second dataset was used to train a positive/negative Naïve Bayes classifier, and consisted of the tweets from the Sanders corpus labeled positive or negative. Thus, as can be seen from Table 1, 1028 tweets were subjective while 2083 were objective. Also, as Table 2 shows, 484 of the 1028 subjective Tweets were positive, while 544 were negative.

\subsection{Microsoft Corpus}

The Microsoft corpus collected for this study was downloaded directly from the Twitter API. This dataset was collected in two stages. First, using the Python library Tweepy (http://tweepy.github.com/), the social networks of four Microsoft-sponsored Twitter accounts were crawled:@technet,@windevs,@VisualStudio, and @Silverlight. These four accounts are used by Microsoft to communicate with information technology professionals and developers, and were chosen because they had relatively large numbers of followers but could still be crawled in a timely manner within the rate limits of the Twitter API. All followers and friends of these accounts who were following less than 600 others were collected, creating the social network for each of the four accounts similar to the visualization in Figure 1. The limit of following 600 users was imposed similarly to [7] as a means of de-noising and limiting the size of the crawled network. With this limitation in place, the number of users crawled for each of the four accounts is displayed in Table 3.

The second stage of data collection involved capturing tweets created by the collected users. This data was harvested using the Java library Twitter4j

(http://twitter4j.org/) in conjunction with the Twitter streaming API. Between January 2, 2013 and February 2, 2013, a total of 2,061,789 tweets were collected from the networks of the Microsoft accounts described above. This portion of the dataset provided the additional features described in Section 3 that were used to enhance community detection on the four Microsoft networks.

\subsection{Combined Datasets}

To ensure significant training data was available for sentiment analysis, a portion of the tweets collected in stage two above were withheld as a training set. A total of 3000 English tweets were randomly selected and removed from the full set of tweets such that they were proportional to the number of tweets collected from each

Table 1. Sanders dataset: subjective and objective tweets.

\begin{tabular}{ccc}
\hline Subjective & Objective & Total \\
\hline 1028 & 2083 & 3111 \\
\hline
\end{tabular}

Table 2. Sanders dataset: subjective tweets.

\begin{tabular}{ccc}
\hline Positive & Negative & Total \\
\hline 484 & 544 & 1028 \\
\hline
\end{tabular}

Table 3. Users crawled for Microsoft accounts.

\begin{tabular}{cccc}
\hline @technet & @windevs & @VisualStudio & @Silverlight \\
\hline 1382 & 15,559 & 26,775 & 18,630 \\
\hline
\end{tabular}


of the four accounts. This set of 3000 tweets was then manually labeled as "positive”, “negative”, or “objecttive” according to the sentiment they expressed.

After this was completed, these tweets were split into two datasets just like the Sanders Corpus, with one set of tweets containing the labels "subjective" and "objective" and the other containing the labels "positive" and "negative". These two datasets are described in Tables $\mathbf{4}$ and $\mathbf{5}$, respectively. Finally, the datasets described in Tables 6 and 7 were created for training the two Naïve Bayes classifiers, combining the tweets from the Sanders Corpus and the training tweets from the Microsoft Corpus.

The remaining Microsoft tweets were then grouped according to the account with which their author was associated as a friend or follower, and further divided by the day on which they were created. Combined with the social networks created from stage one of the Microsoft Corpus collection described in Section 2.2, these tweets created input for the enhanced community detection described in Section 3.

\section{Methods}

Once data had been collected, initial community detection was performed on the friend/follower networks of all four Twitter accounts in the dataset. After this was completed, sentiment, hashtag, reply, mention and retweet features were computed for each day's data and integrated into the community detection process. This section describes the procedures used to accomplish these tasks, focusing particularly on sentiment analysis.

\subsection{Network Construction and Basic Community Detection}

To perform community detection on the friend and follower networks, representations of these networks were created as directed graphs with weighted edges. Edges were created according to the friend and follower rela-

Table 4. Microsoft dataset: subjective and objective tweets.

\begin{tabular}{ccc}
\hline Subjective & Objective & Total \\
\hline 940 & 2060 & 3000 \\
\hline
\end{tabular}

Table 5. Microsoft dataset: positive and negative tweets.

\begin{tabular}{ccc}
\hline Positive & Negative & Total \\
\hline 595 & 345 & 940 \\
\hline
\end{tabular}

Table 6. Combined dataset: subjective and objective tweets.

\begin{tabular}{ccc}
\hline Subjective & Objective & Total \\
\hline 1968 & 4143 & 6111 \\
\hline
\end{tabular}

Table 7. Combined dataset: positive and negative tweets.

\begin{tabular}{ccc}
\hline Positive & Negative & Total \\
\hline 1079 & 889 & 1968 \\
\hline
\end{tabular}

tionships within the four networks, and assigned a weight value of one. This resulted in the networks described in Table 8. The number of vertices in each network was equivalent to the number of accounts in Table 3 . The number of edges, however, was much higher, as every Twitter account was associated with many connections to other individuals. Crawled from the@VisualStudio account, the largest network had had 258,538 edges, while the smallest, from the @technet account, contained only 4834 edges. After the networks had been constructed, both the Infomap and SLPA algorithms were run to perform initial community detection before any additional features were added.

\subsection{Updating the Networks with Additional Features}

Three types of features were used to augment the results of SLPA and Infomap on the initial friend/follower networks. These included: replies, mentions and retweets; hashtags; and sentiment classification of tweets. These features were computed for all of the 32 days in the dataset. Then, they were used to iteratively increment edge weights in the four social networks, and community detection was repeated on the networks using edge weights updated with each day's data. Variations of this technique were attempted in order to determine optimal performance, such as cumulatively maintaining edge weight updates or resetting the network to the initial friend/follower network after computing communities with each day's data.

\subsubsection{Reply, Mention, Retweet, and Hashtag Features}

The first and most intuitive feature included as a supplementary feature for community detection was the presence of replies, mentions, and retweets in tweets referencing other users. The Twitter API conveniently encodes this information in the "entities" section of the data it returns describing tweets, trivializing the extraction of these features. Whenever a reply, mention, or retweet referencing another user in the social network was found in a given day's data, the weight of the edge from the mentioning user to the one mentioned was incremented by one (assuming an edge from the first to the second user existed).

The second supplementary feature for community detection was the presence of hashtags in tweets. Whenever two users mentioned the same hashtag in one or more of their tweets from a given day, the weights of any existing edges between those two users were incremented by one. 
Table 8. Networks created from the Microsoft dataset.

\begin{tabular}{ccc}
\hline Account & Vertices & Edges \\
\hline @Technet & 1382 & 4834 \\
@Windevs & 15,559 & 65,718 \\
@VisualStudio & 26,775 & 258,538 \\
@Silverlight & 18,630 & 127,983 \\
\hline
\end{tabular}

Again, this process was fairly trivial, as Twitter's API also encodes the hashtags used in each tweet in the "entities” section of the metadata describing tweets.

\subsubsection{Sentiment Features}

While calculating the features described above was relatively straightforward, computing sentiment features was much more involved. This section describes the steps used to train the subjective/objective (SO) and positive/negative (PN) Naïve Bayes classifiers, the accuracy achieved on the training set with these classifiers, and the integration of sentiment features with community detection.

The first step towards training both the SO and PN Naïve Bayes classifiers was converting tweet text into a set of features suitable for input to the appropriate classifier. Both unigram and bigram features were used to train each classifier, with bigram features helping to account for cases when words in a sentence were negated (i.e. preceded by the word “not”).

Before unigram and bigram features were created from tweets, tweet text was first preprocessed according to techniques inspired by a previous study [25]. First, all characters in the tweet were converted to lowercase. Then, all hashtags were replaced with "twitterhashtag", retweet designations ("RT") were removed, and usernames were replaced with "twitterusername”. Similarly, URLs were replaced with "twitterurl". Then, tweet text was split into individual word tokens, from which a list of unigram and bigram features was created. While these procedures were applied for both the SO and PN classifiers, additional preprocessing was found to improve the accuracy of the PN classifier. For this classifier, repeated punctuation was replaced with the punctuation symbol and a plus sign (i.e. "!!!” would be replaced with “!+”). Additionally, sentence punctuation following words was split into separate individual tokens, and non-sentence punctuation (such as parenthesis and quotation marks) was removed. Stopwords from the NLTK Stopwords Corpus (with the exception of the tokens "don", "no", "s", "t", "not" and "nor") were also removed from the set of tokens representing each tweet for the PN classifier.

Once preprocessing was completed, the combined SO and PN datasets from Section 2.3 were used to train the NLTK Naïve Bayes classifiers. Ten-fold cross-validation on the training data was used with both classifiers to ap- proximate their accuracy. The SO classifier achieved an average accuracy of $70 \%$ across the ten folds, while the average accuracy of the PN classifier was $79 \%$. The top 20 most informative features as selected by the two Naïve Bayes classifiers are shown in Tables 9 and 10, along with their associated labels. Note that, in both of these tables, bigram features are displayed as two tokens enclosed in parenthesis. Several of the tokens identified as most informative in both sets of features are highly domain-specific. This is due to the fact that the Sanders Corpus focuses particularly on tweets relating to Apple and Android.

With construction of the SO and PN classifiers complete, sentiment features were added as supplementary features to community detection. Similarly to the previous two types of supplementary features, sentiment features were used to increment edge weights within the social network, which in turn influenced the performance of the Infomap and SLPA community detection algorithms.

Several steps were involved with updating edge weights based on sentiment. First, each tweet in a given day's data was classified as either subjective or objective. Then, any tweet classified as objective was further classified as positive or negative. Once sentiment classification was complete, hashtags were used to ensure senti-

Table 9. Top 20 most informative subjective/objective features.

\begin{tabular}{cc}
\hline Feature & Label \\
\hline fucking & subjective \\
(ios, 5) & subjective \\
liked & subjective \\
(customer, service) & subjective \\
(twitterusername, video) & subjective \\
$:($ & subjective \\
totally & subjective \\
birthday & subjective \\
usage & subjective \\
(can't, wait) & subjective \\
(twitterusername, ios) & subjective \\
$:-)$ & subjective \\
phone! & subjective \\
wtf & subjective \\
awesome! & subjective \\
(twitterusername, thanks) & subjective \\
customer & subjective \\
itunes & subjective \\
followers & objective \\
trouble & subjective \\
\hline
\end{tabular}


Table 10. Top 20 most informative positive/negative features.

\begin{tabular}{cc}
\hline Feature & Label \\
:) & positive \\
hate & negative \\
;) & positive \\
awesome & positive \\
itunes & negative \\
issues & negative \\
:-) & positive \\
(?, twitterhashtag) & negative \\
won't & negative \\
fuck & negative \\
sandwich & positive \\
fucking & negative \\
sucks & negative \\
(ice, cream) & positive \\
cream & positive \\
battery & negative \\
issue & negative \\
else & negative \\
(?, twitterusername) & negative \\
(cream, sandwich) & positive \\
\hline
\end{tabular}

ment about unrelated topics was not used to update the network. Thus, edge weights in the network were updated as follows: whenever two users posted a tweet with the same sentiment classification containing the same hashtag, the weights of any edges connecting those users were incremented by one. This effectively could have allowed one tweet to cause two edge updates between two users. If a user's tweet was classified as subjective and assigned the same subjective label (positive or negative) as another user who tweeted the same subjective sentiment and a common hashtag, two edge updates would be made: one for the subjective classification and another for the shared positive or negative classification.

\subsection{Enhanced Community Detection and Sentiment Analysis}

To enhance the basic community detection described in Section 3.1, the three supplementary feature types were used to cumulatively update edge weights in the social network. The social networks for each Microsoft account were updated according to the features in their tweets from each day in the dataset, and community detection using both SLPA and Infomap was performed again after features from each day's data had been included.

As the network was updated community detection was repeated, and the calculated features and detected com- munities were stored for further analysis. This provided for in-depth analysis of sentiment information uncovered when calculating sentiment features for the network as described in Section 4.

\section{Results and Analysis}

The three types of supplementary features significantly increased modularity in the Infomap output for three of the four Microsoft communities. This section describes and analyzes the results of community detection on these networks and shows how the sentiment features computed to enhance community detection provided even more insight when paired with community detection results. In this way, it is shown that community detection and sentiment analysis can be mutually supportive, each providing information to enhance the other.

\subsection{Community Detection}

While community detection was performed using both the Infomap and SLPA algorithms, best results were achieved using Infomap with cumulatively maintained edge updates from each day's data. Thus, in Table 11, community counts, modularity values, and degree ratios for the Infomap algorithm are given (abbreviated in three of the four columns as "C.", "M.", and "D.R." respecttively). The 33 rows are shown for the initial community partitions computed by Infomap for all four Microsoft accounts and for each of the 32 days in the dataset. As can be seen from the data in Table 11, modularity values increased significantly for the@technet, @windevs, and @ Silverlight accounts from the initial network to the final February 2 network. The greatest increase in modularity was in the network from the@windevs account, with the modularity value for the partitioning increasing from 0.1839 to 0.3129 . This suggests that more meaningful communities were uncovered by updating network edge weights with the three supplementary feature types. Increasing weight values of edges connecting nodes with common features allowed community partitions that were likely more representative of the real world interactions in these networks to be discovered.

Interestingly, unlike the other three accounts, the modularity values for the @VisualStudio account did not noticeably increase when the supplementary features were used. While the reason for this is not immediately clear, there are several possible explanations for these results. The@VisualStudio network is significantly larger than any of the other networks, with twice as many edges as the next largest network tested. Furthermore, the average degree of nodes in the @VisualStudio network, 9.66, is much higher than any of the other networks (the next highest, from the @ Silverlight network, is 6.87). Thus, considering the relatively large number of edges in the@VisualStudio network, updates to edge weights 
Table 11. Community detection results.

\begin{tabular}{|c|c|c|c|c|c|c|c|c|c|c|c|c|}
\hline \multirow{2}{*}{ Date } & \multicolumn{3}{|c|}{ @technet } & \multicolumn{3}{|c|}{ @windevs } & \multicolumn{3}{|c|}{ @VisualStudio } & \multicolumn{3}{|c|}{ @Silverlight } \\
\hline & Communities & Modularity & Degree Ratio & C. & $M$ & $D . R$. & C. & M. & $D . R$. & $C$. & M. & $D . R$. \\
\hline Initial & 51 & 0.3462 & 0.4891 & 532 & 0.1839 & 0.4006 & 1141 & 0.3144 & 0.3357 & 445 & 0.2377 & 0.4131 \\
\hline 2-Jan & 51 & 0.3446 & 0.4889 & 536 & 0.1870 & 0.3992 & 1146 & 0.3107 & 0.3349 & 428 & 0.2385 & 0.4203 \\
\hline 3-Jan & 52 & 0.3523 & 0.4848 & 548 & 0.1879 & 0.3946 & 1142 & 0.2985 & 0.3346 & 579 & 0.3180 & 0.3722 \\
\hline 4-Jan & 51 & 0.3632 & 0.4838 & 538 & 0.1967 & 0.3974 & 1145 & 0.2961 & 0.3348 & 587 & 0.3216 & 0.3677 \\
\hline 5-Jan & 51 & 0.3597 & 0.4863 & 549 & 0.2036 & 0.3937 & 1118 & 0.2967 & 0.3376 & 574 & 0.3243 & 0.3709 \\
\hline 6-Jan & 52 & 0.3612 & 0.4834 & 543 & 0.2061 & 0.3938 & 1133 & 0.2933 & 0.3355 & 591 & 0.3270 & 0.3671 \\
\hline 7-Jan & 53 & 0.3652 & 0.4716 & 543 & 0.2112 & 0.3956 & 1141 & 0.3004 & 0.3352 & 596 & 0.3283 & 0.3649 \\
\hline 8-Jan & 51 & 0.3705 & 0.4853 & 563 & 0.2209 & 0.3891 & 1134 & 0.2992 & 0.3335 & 594 & 0.3309 & 0.3629 \\
\hline 9-Jan & 52 & 0.3860 & 0.4776 & 561 & 0.2257 & 0.3889 & 1135 & 0.2978 & 0.3332 & 603 & 0.3319 & 0.3608 \\
\hline 10-Jan & 52 & 0.3806 & 0.4878 & 559 & 0.2333 & 0.3888 & 1147 & 0.3012 & 0.3319 & 593 & 0.3333 & 0.3657 \\
\hline 11-Jan & 53 & 0.3886 & 0.4782 & 554 & 0.2396 & 0.3902 & 1147 & 0.2893 & 0.3289 & 603 & 0.3394 & 0.3621 \\
\hline 12-Jan & 52 & 0.3932 & 0.4827 & 561 & 0.2418 & 0.3883 & 1121 & 0.2925 & 0.3336 & 625 & 0.3376 & 0.3548 \\
\hline 13-Jan & 52 & 0.3903 & 0.4804 & 562 & 0.2455 & 0.3893 & 1117 & 0.3035 & 0.3353 & 614 & 0.3416 & 0.3569 \\
\hline 14-Jan & 54 & 0.3926 & 0.4670 & 567 & 0.2480 & 0.3883 & 1136 & 0.2998 & 0.3324 & 623 & 0.3371 & 0.3528 \\
\hline 15-Jan & 55 & 0.3883 & 0.4644 & 559 & 0.2539 & 0.3899 & 1145 & 0.3000 & 0.3290 & 620 & 0.3398 & 0.3556 \\
\hline 16-Jan & 54 & 0.4106 & 0.4707 & 557 & 0.2662 & 0.3923 & 1153 & 0.3013 & 0.3303 & 624 & 0.3369 & 0.3516 \\
\hline 17-Jan & 55 & 0.4019 & 0.4708 & 559 & 0.2688 & 0.3915 & 1153 & 0.3047 & 0.3290 & 628 & 0.3420 & 0.3522 \\
\hline 18-Jan & 54 & 0.4024 & 0.4640 & 547 & 0.2720 & 0.3932 & 1128 & 0.3096 & 0.3318 & 621 & 0.3443 & 0.3529 \\
\hline 19-Jan & 57 & 0.4023 & 0.4568 & 564 & 0.2734 & 0.3894 & 1144 & 0.3089 & 0.3305 & 630 & 0.3504 & 0.3518 \\
\hline 20-Jan & 56 & 0.4061 & 0.4639 & 564 & 0.2719 & 0.3878 & 1137 & 0.3103 & 0.3302 & 614 & 0.3449 & 0.3542 \\
\hline 21-Jan & 57 & 0.4078 & 0.4568 & 567 & 0.2737 & 0.3891 & 1158 & 0.3097 & 0.3276 & 621 & 0.3542 & 0.3548 \\
\hline 22-Jan & 57 & 0.4093 & 0.4579 & 553 & 0.2893 & 0.3941 & 1159 & 0.3128 & 0.3274 & 634 & 0.3574 & 0.3546 \\
\hline 23-Jan & 59 & 0.4077 & 0.4522 & 567 & 0.2886 & 0.3898 & 1142 & 0.3141 & 0.3298 & 644 & 0.3559 & 0.3490 \\
\hline 24-Jan & 58 & 0.4141 & 0.4561 & 562 & 0.2935 & 0.3923 & 1172 & 0.2985 & 0.3255 & 636 & 0.3566 & 0.3497 \\
\hline 25-Jan & 59 & 0.4150 & 0.4510 & 578 & 0.2950 & 0.3870 & 1168 & 0.3007 & 0.3250 & 639 & 0.3557 & 0.3505 \\
\hline 26-Jan & 58 & 0.4151 & 0.4560 & 572 & 0.2989 & 0.3901 & 1165 & 0.3017 & 0.3245 & 644 & 0.3555 & 0.3499 \\
\hline 27-Jan & 58 & 0.4145 & 0.4548 & 561 & 0.3055 & 0.3917 & 1176 & 0.3028 & 0.3237 & 625 & 0.3607 & 0.3523 \\
\hline 28-Jan & 58 & 0.4186 & 0.4558 & 570 & 0.3045 & 0.3892 & 1178 & 0.3060 & 0.3234 & 629 & 0.3631 & 0.3548 \\
\hline 29-Jan & 59 & 0.4192 & 0.4518 & 580 & 0.3048 & 0.3850 & 1160 & 0.3073 & 0.3249 & 645 & 0.3631 & 0.3492 \\
\hline 30-Jan & 60 & 0.4260 & 0.4507 & 574 & 0.3038 & 0.3882 & 1221 & 0.3210 & 0.3164 & 646 & 0.3577 & 0.3479 \\
\hline 31-Jan & 59 & 0.4281 & 0.4524 & 573 & 0.3096 & 0.3866 & 1161 & 0.3163 & 0.3251 & 665 & 0.3604 & 0.3434 \\
\hline 1-Feb & 60 & 0.4294 & 0.4503 & 564 & 0.3116 & 0.3897 & 1171 & 0.3177 & 0.3230 & 652 & 0.3610 & 0.3480 \\
\hline 2-Feb & 60 & 0.4287 & 0.4501 & 577 & 0.3129 & 0.3853 & 1182 & 0.3163 & 0.3215 & 659 & 0.3615 & 0.3428 \\
\hline
\end{tabular}

based on the three supplementary features may not have been sufficient to significantly alter the community detection output.

Also, while modularity tended to increase with the inclusion of additional features, the degree ratio values decreased as edge weights were updated. While this may at first seem counterintuitive, it is actually to be expected. The modularity metric takes edge weights into account, while the degree ratio does not. Thus, since the number of distinct communities detected increased as additional days' features were included (while maintaining the same internal edge structure), the degree ratio scores decreased.

\subsection{Sentiment Analysis}

As demonstrated in Section 4.1, the three types of supplementary features helped discover communities exhibiting stronger real-world interaction. However, just as sentiment analysis helped facilitate enhanced community detection, community detection also served to enhance 
sentiment analysis. Correlating sentiment information with detected communities permits more in-depth analysis of sentiment information from the level of entire networks down to single communities.

Sentiment analysis data was computed and stored as a part of the community detection process for each of the four Microsoft accounts, which simplified further analysis of this data. In Figure 2, sentiment statistics are presented for each Microsoft account over the 32 days in the dataset. Each of the four curves shown in these figures represents a percentage of the total tweets from each day classified as subjective, objective, positive, or negative. As can be seen from each of these figures, the majority of tweets from each of these networks were classified as objective, with the @technet network exhibiting the highest ratio of objective (average of 60\% per day) to subjective (average of $40 \%$ per day) tweets. In three of the four networks, the percentages of positive and negative tweets were relatively equal. In the @windevs network, however, the objective tweets were primarily negative. Of the objective tweets produced by this network, $44 \%$ per day on average were positive, while $56 \%$ were classified as negative.

While looking at the overall sentiment trends for each of these accounts is interesting, this information is ultimately of limited usefulness. A multitude of topics are discussed by many different groups of people in each of these networks. Realistically, it would be much more helpful to consider sentiment expressed by specific groups of people or about specific topics.

To facilitate a more narrowed analysis, popular tweet topics in the dataset were identified using hashtags.
Table 12 shows the top ten most popular hashtags appearing in the entire dataset and the number of times each was used. Four out of these ten hashtags ("windowsphone”, “wpdev”, “wp”, and “wp8”) reference Microsoft's smartphone platform, Windows Phone, indicating that this was a popular topic during the period the dataset was collected. Thus, to demonstrate the power of combining sentiment analysis with community detection, Windows Phone was chosen as a topic for deeper analysis.

To understand overall sentiment towards Windows Phone, sentiment classifications were tallied for tweets containing the above four hashtags from the networks associated with each Microsoft account. Figure 3 shows percentages of tweets pertaining to Windows Phone expressing each of the four sentiment labels from one of the Microsoft networks,@windevs. In the@windevs network, the majority of tweets from each day pertaining to Windows phone were classified as objective (73\% on average), and of the subjective tweets there was a fairly even split of positively (12\% on average) and negatively (14\% on average) classified tweets. Figure 3 represents sentiment scores pertaining to the entire@windevs network. But, by combining community detection and sentiment analysis results, an even more granular perspective becomes available. Figure 4 displays Windows Phone-related sentiment classification percentages for the largest detected community in the@windevs network, and reveals a significantly different trend than that of the @windevs network as a whole. While the majority of tweets produced by this community are still objective (an average of $73 \%$ per day), of the subjectively classified
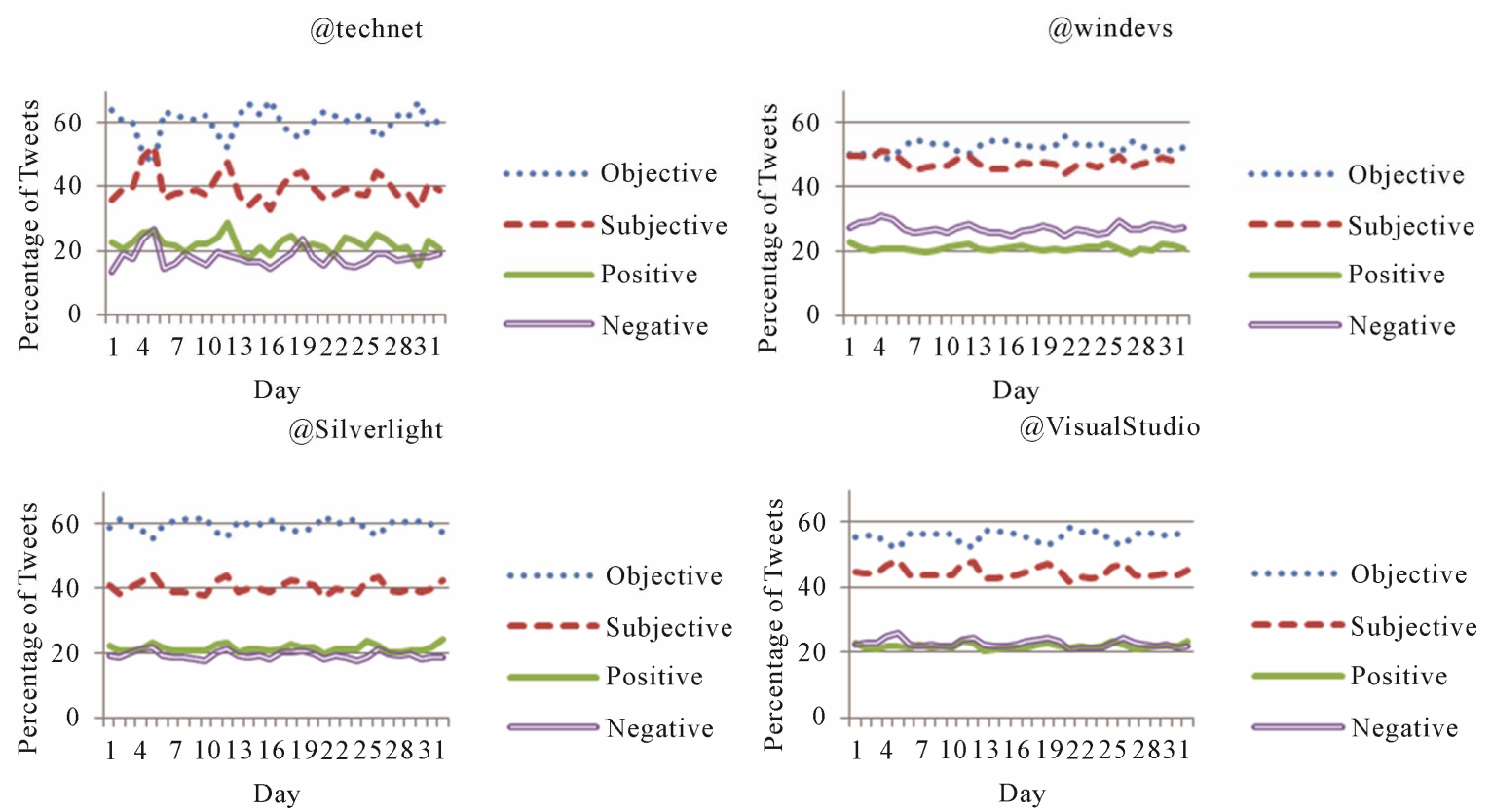

Figure 2. Overall sentiment statistics for Microsoft networks. 


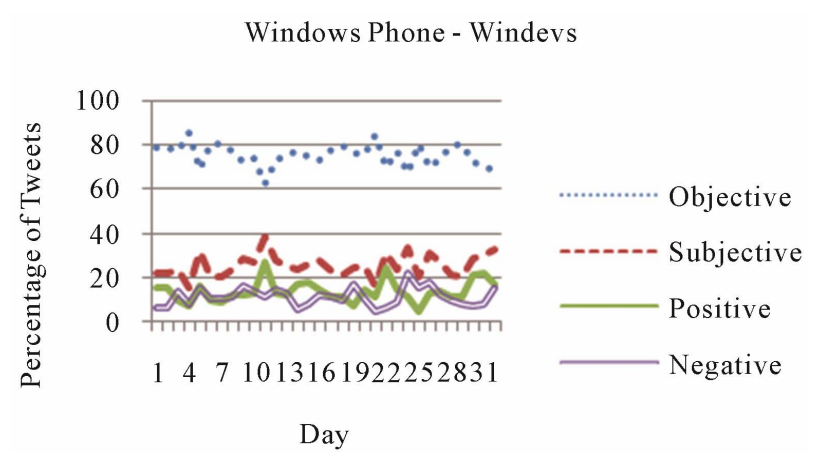

Figure 3. Windows phone sentiment-@windevs.

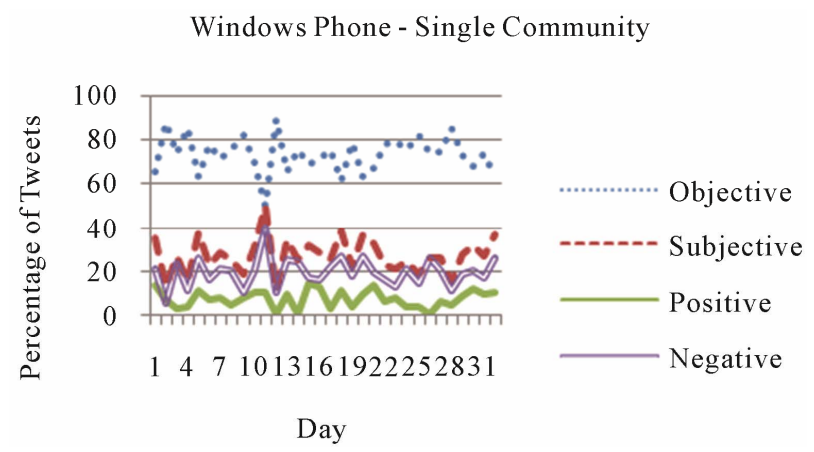

Figure 4. Windows Phone sentiment-largest community from@windevs.

Table 12. Top ten hashtags.

\begin{tabular}{cc}
\hline Hashtag & Count \\
\hline tech & 6892 \\
windows8 & 5463 \\
microsoft & 4871 \\
windowsphone & 4188 \\
wpdev & 3788 \\
wp & 3332 \\
wp8 & 2906 \\
getglue & 2407 \\
android & 2260 \\
fb & 2258 \\
\hline
\end{tabular}

tweets only $7 \%$ per day on average are positive, while $19 \%$ on average are negative. Thus, the largest community detected within the @windevs network exhibited significantly more negative sentiment towards Windows Phone than the network as a whole. This demonstrates how community data was able to enhance sentiment analysis by permitting a more granular view of sentiment from a specific community within the larger@windevs network.

\section{Conclusions}

Due to the rising popularity of online social networks, analysis of OSNs has become the focus of many recent research efforts. Two common topics are community detection and sentiment analysis, which examine the structure and content of social networks. Though community detection and sentiment analysis are usually treated as separate issues, this research integrates the two and demonstrates how these techniques can be used to enhance each other.

The publicly available Sanders Sentiment Corpus was used to provide data for this study, in addition to four Microsoft-related social networks downloaded directly from the Twitter API. While the Sanders Corpus was relatively small, the Microsoft dataset was significantly larger. Overall, the combined Microsoft dataset contained the friend and follower networks of 62,346 Twitter users and 2,061,789 of their tweets, collected over a period of 32 days.

Community detection was performed on the friend/ follower networks of the four Microsoft accounts using the SLPA and Infomap algorithms. This community detection was then enhanced with three types of additional features: replies, mentions, and retweets; hashtags; and sentiment classifications. The sentiment classifications were derived using two Naïve Bayes classifiers trained with the Sanders dataset and a small portion of handlabeled tweets from the Microsoft dataset. These three feature types were calculated from each day's tweets, and were applied to the four networks in the dataset by increasing edge weights between network nodes.

Using the three supplementary feature types to enhance community detection improved modularity values in the Infomap output on three of the four networks studied. The most dramatic change in modularity was in the @ windevs network, with modularity increasing from 0.1839 to 0.3129 . Furthermore, combining sentiment classifications and community groupings permitted more in-depth analysis of sentiment data from the same @ windevs network, which was illustrated by examining sentiment directed towards Microsoft's Windows Phone. Thus, this study takes the novel approach of combining community detection and sentiment analysis, demonstrating that these techniques can be used in a mutually informative way with each enhancing the other.

\section{Acknowledgements}

We would like to thank Houghton College for providing funding and technical resources for this research.

\section{REFERENCES}

[1] A. H. Wang, "Don't Follow Me: Spam Detection in Twitter," Proceedings of the 2010 International Conference on Security and Cryptography (SECRYPT), Piraeus, 26-28 July 2010, pp. 1-10.

[2] T. Falkowski, A. Barth and M. Spiliopoulou, "Studying 
Community Dynamics with an Incremental Graph Mining Algorithm," Proceedings of the 14th Americas Conference on Information Systems (AMCIS 2008), Toronto, 14-17 August 2008, pp. 1-11.

[3] K. Liu, W. Li and M. Guo, "Emoticon Smoothed Language Models for Twitter Sentiment Analysis," Proceedings of the 26th AAAI Conference on Artificial Intelligence, Toronto, 22-26 July 2012, pp. 1678-1684.

[4] A. Burns and B. Eltham, "Twitter Free Iran: An Evaluation of Twitter's Role in Public Diplomacy and Information Operations in Iran's 2009 Election Crisis,” Record of the Communications Policy and Research Forum 2009, Sydney, 19-20 November 2009, pp. 322-334.

[5] D. Gayo-Avello, P. T. Metaxas and E. Mustafaraj, "Limits of Electoral Predictions Using Twitter," Proceedings of the International Conference on Weblogs and Social Media (ICWSM) 2011, Barcelona, 17-21 July 2011, pp. 490493.

[6] B. J. Jansen, M. Zhang, K. Sobel and A. Chowdury, "Twitter Power: Tweets as Electronic Word of Mouth," Journal of the American Society for Information and Technology, Vol. 60, No. 11, 2009, pp. 2169-2188. doi:10.1002/asi.21149

[7] M. van Meeteren, A. Poorthuis and E. Dugundji, "Mapping Communities in Large Virtual Social Networks," Proceedings of the 1st International Forum on the Application and Management of Personal Electronic Information, Cambridge, 12-13 October 2009, 8 Pages.

[8] S. E. Schaeffer, "Graph Clustering," Computer Science Review, Vol. 1, No. 1, 2007, pp. 27-64. doi:10.1016/j.cosrev.2007.05.001

[9] M. Girvan and M. E. J. Newman, "Community Structure in Social and Biological Networks," Proceedings of the National Academy of the Sciences of the United States of America, Vol. 99, No. 12, 2002, pp. 7821-7826. doi:10.1073/pnas.122653799

[10] I. X. Y. Leung, P. Hui, P. Lio and J. Crowcroft, "Towards Real-Time Community Detection in Large Networks," Physical Review E, Vol. 79, No. 6, 2009, Article ID: 066107. doi:10.1103/PhysRevE.79.066107

[11] T. Falkowski, A. Barth and M. Spilioupoulou, "Dengraph: A Density-Based Community Detection Algorithm," Proceedings of the IEEE/WIC/ACM International Conference on Web Intelligence, Silicon Valley, 2-5 November 2007, pp. 113-115.

[12] M. E. J. Newman and M. Girvan, "Finding and Evaluating Community Structure in Networks," Physical Review E, Vol. 69, No. 2, 2004, Article ID: 026113. doi:10.1103/PhysRevE.69.026113

[13] S. Fortunato and M. Barthelemy, "Resolution Limit in Community Detection," Proceedings of the National Academy of Sciences of the United States of America, Vol. 104, No. 1, 2007, pp. 36-41. doi:10.1073/pnas.0605965104

[14] B. H. Good, Y. de Montjoye and A. Clauset, "Perform- ance of Modularity Maximization in Practical Contexts," Physical Review E, Vol. 81, No. 4, 2010, Article ID: 046106. doi:10.1103/PhysRevE.81.046106

[15] A. Lancichinetti, F. Radicchi, J. J. Ramasco and S. Fortunato, "Finding Statistically Significant Communities in Networks,” PLoS ONE, Vol. 6, No. 4, 2011, Article ID: e18961. doi:10.1371/journal.pone.0018961

[16] A. Lancichinetti, S. Fortunato and J. Kertész, "Detecting the Overlapping and Hierarchical Community Structure in Complex Networks," New Journal of Physics, Vol. 11, No. 3, 2009, Article ID: 033015. doi:10.1088/1367-2630/11/3/033015

[17] M. Newman, "Networks: An Introduction,” 1st Edition, Oxford University Press, Inc., New York, 2010.

[18] J. Xie, "Agent-Based Dynamics Models for Opinion Spreading and Community Detection in Large-Scale Social Networks,” Ph.D. Thesis, Rensselaer Polytechnic Institute, Troy, 2012.

[19] M. Rosvall and C. T. Bergstrom, "Maps of Random Walks on Complex Networks Reveal Community Structure," Proceedings of the National Academy of the Sciences of the United States of America, Vol. 105, No. 4, 2008, pp. 1118-1123. doi:10.1073/pnas.0706851105

[20] A. Esuli and F. Sebastiani, "SENTIWORDNET: A Publicly Available Lexical Resource for Opinion Mining," Proceedings of the 5th Conference on Language Resources and Evaluation, Genoa, 24-26 May 2006, pp. 417-422.

[21] A. Hamouda and M. Rohaim, "Reviews Classification Using SentiWordNet Lexicon," The Online Journal on Computer Science and Information Technology, Vol. 2, No. 1, 2011, pp. 120-123.

[22] F. Chaumartin, "UPAR7: A Knowledge-Based System for Headline Sentiment Tagging," Proceedings of the 4th International Workshop on Semantic Evaluations, Prague, 23-24 June 2007, pp. 422-425. doi:10.3115/1621474.1621568

[23] K. Denecke, "Using SentiWordNet for Multilingual Sentiment Analysis," Proceedings of the IEEE 24th International Conference on Data Engineering Workshop, Cancún, 7-12 April 2008, pp. 507-512.

[24] B. Ohana and B. Tierney, "Sentiment Classification of Reviews Using SentiWordNet," Proceedings of the 9th IT\&T Conference, Dublin, 22-23 October 2009, 9 Pages.

[25] A. Pak and P. Paroubek, "Twitter As a Corpus for Sentiment Analysis and Opinion Mining," Proceedings of the International Conference on Language Resources and Evaluation, Malta, 19-21 May 2010, pp. 1320-1326.

[26] M. Speriosu, N. Sudan, S. Upadhyay and J. Baldridge, "Twitter Polarity Classification with Label Propagation Over Lexical Links and the Follower Graph,” Proceedings of the 1st Workshop on Unsupervised Learning in NLP, Edinburgh, 30 July 2011, pp. 53-63. 